Title : will be set by the publisher

Editors : will be set by the publisher

EAS Publications Series, Vol. ?, 2013

\title{
RESTORATION OF HYPERSPECTRAL ASTRONOMICAL DATA WITH SPECTRALLY VARYING BLUR
}

\author{
F. Soulez ${ }^{1}$, E. Thiébaut ${ }^{1}$ and L. Denis ${ }^{2}$
}

\begin{abstract}
In this paper we present a method for hyper-spectral image restoration for integral field spectrographs (IFS) data. We specifically address two topics: (i) the design of a fast approximation of spectrally varying operators and (ii) the comparison between two kind of regularization functions: quadratic and spatial sparsity functions. We illustrate this method with simulations coming from the Multi Unit Spectroscopic Explorer (MUSE) instrument. It shows the clear increase of the spatial resolution provided by our method as well as its denoising capability.
\end{abstract}

\section{Introduction}

In the last decade, integral field spectrographs (IFS) have become popular tools for astronomical observations. Such instruments are now installed on all the main optical telescope facilities around the world. They provide spatially resolved spectra of a whole region of the sky, yielding $(\boldsymbol{\theta}, \lambda)$ data cubes - with $\boldsymbol{\theta}$ the $2 \mathrm{D}$ angular position and $\lambda$ the wavelength — with several hundreds of wavelength bins. With IFS, astronomical data enters the hyper-spectral era. New dedicated image reconstruction techniques are needed to take full advantage of the data gathered by these instruments. Because the light is split on multiple channels instead of being integrated on a single image, the information contents is increased at the cost of a lower signal to noise for the same exposure time. Furthermore, atmospheric turbulence and instrumental response spatially blur the observations, degrading the spatial resolution.

\footnotetext{
1 Université de Lyon, Lyon, F-69003, France; Université Lyon 1, Observatoire de Lyon, 9 avenue Charles André, Saint-Genis Laval, F-69230, France; CNRS, UMR 5574, Centre de Recherche Astrophysique de Lyon; École Normale Supérieure de Lyon, Lyon, F-69007, France.

${ }^{2}$ Université de Lyon, F-42023, Saint-Etienne, France, CNRS, UMR5516, Laboratoire Hubert Curien, F-42000, Saint-Etienne, France, Université de Saint-Etienne, Jean Monnet, F-42000, Saint-Etienne, France.
} 
Title : will be set by the publisher

First attempts to restore multi-channel images consisted in applying classical 2D restoration techniques like Wiener filter or Richardson-Lucy algorithm on each individual channel. The caveat of these approaches is to ignore the natural spectral correlations present in the data. The first restoration technique specifically dedicated to multichannel data (Hunt and Kubler, 1984) was a Minimum Mean Square Error (MMSE) restoration filter based on the assumption that signal auto-correlation is spatially and spectrally separable. This assumption was later relaxed (Galatsanos and Chin, 1989) and many other multichannel linear restoration filters have been proposed since (Tekalp and Pavlovic, 1990; Galatsanos et al., 1991; Katsaggelos et al., 1993; Gaucel et al., 2006). More recently, Fourier/wavelet restoration techniques (Neelamani et al., 2004) have been adapted to multispectral data (Benazza-Benyahia and Pesquet, 2006; Duijster et al., 2009). In remote sensing, some authors (Akgun et al., 2005; Bobin et al., 2009) combine demixing and restoration to achieve enhanced spatial resolution given the strong assumption that the observed scene is composed of only a few materials with unknown spectrum.

Most of these developments on restoration of multi-spectral images are dedicated to remote sensing and color (RGB) images. Those methods can't easily be directly applied to astronomical data with its specific features like large dynamic range and strong sharp features (e.g. narrow emission lines or peaked sources). Few restoration techniques for multi-spectral astronomical images have been proposed for $(x, \lambda)$ data (slit spectrography) (Courbin et al., 2000; Lucy and Walsh, 2003) or $(x, y, \lambda)$ data composed of slit spectrography scans (Rodet et al., 2008). However astronomical hyperspectral processing is gaining more and more attention as it is becoming mandatory to fully exploit the capabilities of new integral field spectrographs (e.g. second generation VLT instruments MUSE and KMOS) and restoration algorithms dedicated to IFS begin to appear (Soulez et al., 2008; Bongard et al., 2009; Bongard et al., 2011; Bourguignon et al., 2011a; Bourguignon et al., 2011b).

Following the work we have done in Soulez et al.(2011) and Bongard et al.(2011), we present in this paper a deconvolution method based on a so called inverse problem approach. It is very generic and exploits intrinsic regularities of hyper-spectral data. We suppose that a good estimation of the point spread function (PSF) is provided by other means (e.g. by calibration on the telescope guiding stars or on information from the adaptive optics system) and defer the blind restoration problem to a later time.

Our approach will be illustrated on data provided by the MUSE IFS simulator. Still in integration, the MUSE IFS (Henault et al., 2003) will be installed on the ESO Very Large Telescope (VLT) in 2013. It is a "slicer" based IFS that covers in its wide field mode a $60^{\prime \prime} \times 60^{\prime \prime}$ spectroscopic field-of-view subdivided into a grid of about $300 \times 300$ spatial elements (spaxels). To each spaxel corresponds a spectrum, obtained by dispersing the light on 3463 equally spaced spectral bins from $480 \mathrm{~nm}$ to $930 \mathrm{~nm}$. 


\section{Problem Formulation}

\subsection{Model description}

The direct model describes how the observed data $\boldsymbol{y}$ is related to the 3D intensity distribution of the object of interest $I(\boldsymbol{\theta}, \lambda)$ with $\boldsymbol{\theta}=\left(\theta_{1}, \theta_{2}\right)$ the 2-D position angle. This data cube $\boldsymbol{y}$ is composed of $N_{\lambda}$ monochromatic images of $N_{\Omega}$ pixels. It writes:

$$
y_{\boldsymbol{k}, \ell}=g\left(\boldsymbol{\theta}_{\boldsymbol{k}}, \lambda_{\ell}\right)+e_{\boldsymbol{k}, \ell}
$$

where $g(\boldsymbol{\theta}, \lambda)$ is the distribution sampled by the detector, $\left(\boldsymbol{\theta}_{\boldsymbol{k}}, \lambda_{\ell}\right)$ are the spatiospectral coordinates of the pixel at the 2D spatial index $\boldsymbol{k}$ and spectral index $\ell$, and $e_{\boldsymbol{k}, \ell}$ accounts for the errors (noise and model approximations). The sampled distribution $g(\boldsymbol{\theta}, \lambda)$ writes:

$$
g(\boldsymbol{\theta}, \lambda)=\iiint h\left(\boldsymbol{\theta}-\boldsymbol{\theta}^{\prime}, \lambda-\lambda^{\prime} ; \boldsymbol{\theta}^{\prime}, \lambda^{\prime}\right) I\left(\boldsymbol{\theta}^{\prime}, \lambda^{\prime}\right) \mathrm{d}^{2} \boldsymbol{\theta}^{\prime} \mathrm{d} \lambda^{\prime}
$$

where $h(\Delta \boldsymbol{\theta}, \Delta \lambda ; \boldsymbol{\theta}, \lambda)$ is the recentred PSF at position $\boldsymbol{\theta}$ and at wavelength $\lambda$. In words, the PSF is the linear response of the total observing system (atmosphere + optics + detector) for a monochromatic point-like source at $(\boldsymbol{\theta}, \lambda)$.

At best, we can only recover an approximation of the true object brightness distribution, we choose to represent the sought distribution by:

$$
I(\boldsymbol{\theta}, \lambda)=\sum_{\boldsymbol{k}, \ell} x_{\boldsymbol{k}, \ell} b_{\boldsymbol{k}, \ell}(\boldsymbol{\theta}, \lambda)
$$

where $\boldsymbol{x}$ are the unknown parameters and $b_{\boldsymbol{k}, \ell}(\boldsymbol{\theta}, \lambda)$ are basis functions. Using interpolation functions for the basis functions and the same spatio-spectral sampling for the model and the data yields:

$$
x_{\boldsymbol{k}, \ell} \approx I\left(\boldsymbol{\theta}_{\boldsymbol{k}}, \lambda_{\ell}\right) .
$$

The direct model then writes:

$$
y_{\boldsymbol{k}, \ell}=\sum_{\boldsymbol{k}^{\prime}, \ell^{\prime}} H_{\boldsymbol{k}, \ell, \boldsymbol{k}^{\prime}, \ell^{\prime}} x_{\boldsymbol{k}^{\prime}, \ell^{\prime}}+e_{\boldsymbol{k}, \ell}
$$

with $\mathbf{H}$ the linear operator corresponding to the system response. Using compact matrix notation:

$$
\boldsymbol{y}=\mathbf{H} \cdot \boldsymbol{x}+\boldsymbol{e} .
$$

Under the same assumption as those leading to Eq. (2.4):

$$
H_{\boldsymbol{k}, \ell, \boldsymbol{k}^{\prime}, \ell^{\prime}} \approx h\left(\boldsymbol{\theta}-\boldsymbol{\theta}^{\prime}, \lambda-\lambda^{\prime} ; \boldsymbol{\theta}^{\prime}, \lambda^{\prime}\right) \Pi \boldsymbol{\theta}^{2} \Pi \lambda,
$$

with $\Pi \boldsymbol{\theta}^{2}$ and $\Pi \lambda$ the pixel size and the effective spectral bandwidth respectively.

The linear operator $\mathbf{H}$ models the linear response of the observation system. It can be described by a PSF which varies both spatially and spectrally. As the 
Title : will be set by the publisher

telescope and the atmosphere don't have any effect along the spectral dimension, blur along spectral dimension is only due to the IFS. Conversely, without adaptive optics system, the atmosphere is responsible for most of the blur along spatial dimensions. As the field of view (FOV) is limited, we can assume that this PSF is spatially shift invariant:

$$
h_{\lambda}((\Delta \boldsymbol{\theta}, \Delta \lambda)=h(\Delta \boldsymbol{\theta}, \Delta \lambda ; \lambda)
$$

However wavelength-wise PSF's $h_{\lambda}$ may be centered at a location $\boldsymbol{\theta}_{\lambda}$ which depends on the wavelength so as to account for imperfect instrumental alignment and atmospheric differential refractive index (ADR). Furthermore PSF is not necessarily normalized in order to account for the variable throughput (atmospheric and instrumental transmission). Finally, the operator $\mathbf{H}$ can be described as a spectrally varying convolution.

\subsection{Spectrally varying PSF approximation}

If the observing system is spatially and spectrally shift-invariant, $\mathbf{H}$ is a block Toeplitz matrix with Toeplitz block that can be diagonalized by means of discrete Fourier transforms (under a circulant approximation or providing a proper processing of the egdes as explained later). Such transforms being efficiently computed thanks to the FFT (Fast Fourier Transform) algorithm. In the considered case, the PSF is spatially shift-invariant but depends on the wavelength of the source. In order to implement a fast version of such an operator $\mathbf{H}$ storing the full $\mathbf{H}\left(\geq 10^{12}\right.$ elements) is not possible and, even so, applying it in this form would take to much CPU time, we propose to follow the prescription of Denis et al.(2011) and write:

$$
h_{\lambda}(\Delta \boldsymbol{\theta}, \Delta \lambda) \approx \sum_{p} \phi_{p}(\lambda) h_{p}(\Delta \boldsymbol{\theta}, \Delta \lambda)
$$

where:

$$
h_{p}(\Delta \boldsymbol{\theta}, \Delta \lambda) \stackrel{\text { def }}{=} h\left(\Delta \boldsymbol{\theta}, \Delta \lambda ; \lambda_{p}\right)
$$

are samples at differents wavelengts $\left\{\lambda_{p}\right\}_{p=1}^{P}$ of the recentered spectrally-varying PSF and $\left\{\phi_{p}(\lambda): \mathbb{R} \mapsto \mathbb{R}\right\}_{p=1}^{P}$ are spectral interpolation functions. With this modeling of the PSF, the operator $\mathbf{H}$ becomes:

$$
\mathbf{H}=\sum_{p=1}^{P} \mathbf{H}_{p} \cdot \mathbf{K}_{p}
$$

with $\mathbf{H}_{p}$ the discrete 3D convolution by $h_{p}(\Delta \boldsymbol{\theta}, \Delta \lambda)$ and $\mathbf{K}_{p}$ an operator which extracts a subset of the spectral range (around $\lambda_{p}$ ) and weights the selected spaxels by the interpolation function $\phi_{p}(\lambda)$. Operators $\mathbf{H}_{p}$ are implemented using 3D FFT's while $\mathbf{K}_{p}$ 's are very sparse as their only non-zero coefficients are along their diagonal. Thus, as long as the spectral support of $h_{p}(\Delta \boldsymbol{\theta}, \Delta \lambda)$ is sufficiently small compared to the patch selected by $\mathbf{K}_{p}$, applying $\mathbf{H}$ (or its adjoint $\mathbf{H}^{*}=$ 
$\left.\sum_{p} \mathbf{K}_{p}^{\mathrm{T}} \cdot \mathbf{H}_{p}^{*}\right)$ is very fast. The computations are dominated by the calculus of the FFT.

First order (linear) interpolation with a subset of PSF built by sampling on a uniform grid $\left\{\lambda_{1}, \ldots, \lambda_{P}\right\}$ leads to interpolation weights supported on a patch twice the grid step along. Each patch extracted by $\mathbf{K}_{p}$ are convolved only with the coresponding PSF $h_{p}(\Delta \boldsymbol{\theta}, \Delta \lambda)$. As a consequence, the computational cost for applying our spectrally varying operator is only roughly twice the cost the applying a non-varying operator: (4 versus 2 FFTs). As stated in Denis et al.(2011), such approximation of the shift varying PSF preserves some good properties of the PSF, namely normalization, positivity and symmetry.

\section{Maximum a posteriori approach}

Restoration is a typical ill-posed problem (Bertero and Boccacci, 1998). We choose to solve it by adding priors in a classical Maximum A Posteriori (MAP) framework. This is achieved by estimating the object $\boldsymbol{x}^{+}$that minimizes a cost function $f(\boldsymbol{x})$ :

$$
\begin{aligned}
\boldsymbol{x}^{+} & =\underset{\boldsymbol{x}}{\arg \min } f(\boldsymbol{x}), \\
f(\boldsymbol{x}) & =f_{\text {data }}(\boldsymbol{x})+f_{\text {prior }}(\boldsymbol{x}) .
\end{aligned}
$$

This cost function $f(\boldsymbol{x})$ is the sum of a likelihood penalty $f_{\text {data }}(\boldsymbol{x})$ ensuring the agreement between the model and the data $\boldsymbol{y}$, and a regularization penalty $f_{\text {prior }}(\boldsymbol{x})$ introducing subjective a priori knowledge about the object.

\subsection{Likelihood and Noise Statistics}

Assuming Gaussian noise, the likelihood penalty reads:

$$
f_{\text {data }}(\boldsymbol{x})=[\boldsymbol{y}-\mathbf{H} \cdot \boldsymbol{x}]^{\mathrm{T}} \cdot \mathbf{W}_{\mathrm{err}} \cdot[\boldsymbol{y}-\mathbf{H} \cdot \boldsymbol{x}],
$$

where the weighting matrix $\mathbf{W}_{\text {err }}=\mathbf{C}_{\text {err }}^{-1}$ is the inverse of the angular-spectral covariance of the errors (noise + approximations). Assuming uncorrelated noise, $\mathbf{W}_{\text {err }}$ is diagonal and Eq. (3.3) simplifies to:

$$
f_{\text {data }}(\boldsymbol{x})=\sum_{\boldsymbol{k}, \ell} w_{\boldsymbol{k}, \ell}[\boldsymbol{y}-\mathbf{H} \cdot \boldsymbol{x}]_{\boldsymbol{k}, \ell}^{2}
$$

where $1 / w_{\boldsymbol{k}, \ell}$ is the noise variance of the measurements at pixel $\boldsymbol{k}$ and channel $\ell$. This model can cope with non-stationary noise and can be used to express confidence on each measurements. Since unmeasured data can be considered as having infinite variance, we readily deal with missing or bad pixels as follows:

$$
w_{\boldsymbol{k}, \ell} \stackrel{\text { def }}{=} \begin{cases}\operatorname{Var}\left(y_{\boldsymbol{k}, \ell}\right)^{-1} & \text { if } y_{\boldsymbol{k}, \ell} \text { is measured } \\ 0 & \text { otherwise. }\end{cases}
$$

This treatment of missing data is rigorous because (i) it consistently accounts for unmeasured data and bad pixels, and (ii) it allows to properly expand the 
synthesized FOV to avoid field aliasing and border artifacts caused by convolution using Fourier transform. This formulation provides a rigorous scheme to take into account photons emitted by sources outside the FOV that are measured because of the blurring. As a consequence, restored object has to be estimated even outside of the field of view, by extending the size of the FOV by at least the PSF size. As we showed in Soulez et al.(2008) and Bongard et al.(2011), this may lead to a small extension of the FOV of the instrument that can be relatively significant when this FOV is small like in the SNIFS instrument considered by Bongard et al. (2011).

Except for very low detector noise $\left(<\right.$ few $e^{-}$per pixel), the total noise (Gaussian detector noise plus Poisson noise) is approximated by a non stationary uncorrelated Gaussian noise (Mugnier et al., 2004):

$$
w_{\boldsymbol{k}, \ell} \stackrel{\text { def }}{=} \begin{cases}\left(\gamma \max \left(y_{\boldsymbol{k}, \ell}, 0\right)+\sigma_{\boldsymbol{k}, \ell}^{2}\right)^{-1} & \text { if } y_{\boldsymbol{k}, \ell} \text { is measured } \\ 0 & \text { otherwise }\end{cases}
$$

where $\gamma$ accounts for the quantization factor of the detector (i.e.number of photon per quantization level) and $\sigma_{\boldsymbol{k}, \ell}^{2}$ is the variance on the pixel $(\boldsymbol{k}, \ell)$ of other sources of noise than the signal, like for example read-out noise for instance.

\subsection{Priors}

In our MAP framework, priors on the object are enforced by the regularization penalty $f_{\text {prior }}(\boldsymbol{x})$ term of the total cost function $f(\boldsymbol{x})$. It introduces in the solution generic knowledge about the observed objects. In addition, we enforce strict priors to ensure the non negativity of the parameters $\boldsymbol{x}$.

As in hyper-spectral imaging the spatial and the spectral dimension have different physical meaning we split the regularization function as the sum of a spatial regularization $f_{\text {spatial }}(\boldsymbol{x})$ and a spectral regularization $f_{\text {spectral }}(\boldsymbol{x})$ :

$$
f_{\text {prior }}(\boldsymbol{x})=\alpha f_{\text {spatial }}(\boldsymbol{x})+\beta f_{\text {spectral }}(\boldsymbol{x}) .
$$

where $\alpha$ and $\beta$ are hyper-parameters that have to be tuned to set the importance of the priors.

In this work we propose two kind of regularization functions: (i) a quadratic regularisation and (ii) a spatial sparsity regularisation.

\subsubsection{Quadratic Regularization}

Quadratic regularization (so called Tikhonov) is the most simple prior that can be introduced in our MAP scheme. In that case and with the least square likelihood function defined in 3.3, the minimization of Eq. (3.1) shows good convergence property since the total cost function $f(\boldsymbol{x})$ is strictly convex and quadratic.

As stated in (Bongard et al., 2011), it is customary to minimize the quadratic norm of finite differences to account for continuities along the three dimensions of 
the brightness distribution. The regularization functions are thus:

$$
\begin{aligned}
f_{\text {spatial }}(\boldsymbol{x}) & =\sum_{\boldsymbol{k}, \ell}\left(\nabla_{k_{1}} \boldsymbol{x}\right)^{2}+\left(\nabla_{k_{2}} \boldsymbol{x}\right)^{2}, \\
f_{\text {spectral }}(\boldsymbol{x}) & =\sum_{\boldsymbol{k}, \ell}\left(\nabla_{\ell} \boldsymbol{x}\right)^{2},
\end{aligned}
$$

where $\nabla_{i} \boldsymbol{x}$ is the finite differential operator along the dimension indexed by the letter $i$.

\subsubsection{Spatial only sparsity regularization}

In wide field observations, astronomical data is mainly composed of bright objects

(stars, galaxy) over a flat background. Most of the quite large MUSE field of view will thus contain only background. As a consequence, the observed scene is intrinsically spatially sparse. This spatial sparsity prior can be enforced by means of structured norms (Fornasier and Rauhut, 2008; Kowalski and Torrésani, 2009):

$$
f_{\text {sparsity }}(\boldsymbol{x})=\sum_{\boldsymbol{k}}\left[\sqrt{\sum_{\ell} x_{\boldsymbol{k}, \ell}^{2}+\epsilon^{2}}-\epsilon\right]
$$

where $\epsilon$ is a small real number $\left(\epsilon \approx 10^{-9}\right)$ that ensures the derivability in 0 (hyperbolic approximation of the $\ell_{1}$ norm). This regularization enforces spatial sparsity and spectral correlation since it favors solutions where bright features in each spectral channel are at the same spatial location.

The regularization defined in Eq. (3.9) does not ensure the spectral continuity of the solution whereas in practice the spectral energy distribution (SED) of a pixel should be relatively smooth excepted near emission and absorption lines. For that reason we introduce an additional regularization function:

$$
f_{\text {spectral }}(\boldsymbol{x})=\sum_{\boldsymbol{k}, \ell}\left[\sqrt{\left(\nabla_{\ell} \boldsymbol{x}_{\boldsymbol{k}}\right)^{2}+\zeta^{2}}-\zeta\right] .
$$

This regularization tends to smooth the spectra $x_{\boldsymbol{k}}$ but preserve discontinuities where $\left|\nabla_{\ell} \boldsymbol{x}_{\boldsymbol{k}}\right| \gg \zeta$. This situation is for example encountered at absorption or emission lines, which shall not be smoothed.

\subsubsection{Renormalization}

Owing to the large variations of the dynamical range between spectral channel of astronomical images, these regularizations lead to over-regularize bright features or under-regularize faint ones. For that reason, as Bongard et al.(2011) we rather suggest to apply these regularizations to spectrally whitened object $\boldsymbol{x}^{\prime}$ :

$$
x_{\boldsymbol{k}, \ell}^{\prime}=x_{\boldsymbol{k}, \ell} / s_{\ell}
$$


with $s_{\ell}=\left\langle x_{\boldsymbol{k}, \ell}\right\rangle_{\boldsymbol{k}}$ the spatially averaged object spectrum -\langle\rangle$_{\boldsymbol{k}}$ denotes averaging over pixel index $\boldsymbol{k}$. To avoid introducing more non-linearity in regularizations, we estimate the mean object spectrum directly from the data:

$$
s_{\ell}=\left\langle y_{\boldsymbol{k}, \ell}\right\rangle_{\boldsymbol{k}} / \eta_{\ell}
$$

with $\eta_{\ell}=\eta\left(\lambda_{\ell}\right)$ the effective throughput in $\ell$-th spectral channel:

$$
\eta_{\ell}=\iiint h_{\ell}(\Delta \boldsymbol{\theta}, \Delta \lambda) \mathrm{d}^{2} \Delta \boldsymbol{\theta} \mathrm{d} \Delta \lambda .
$$

\subsection{Algorithm description}

As discussed in section 3.1, due to the convolution process, flux from the object just outside of the field of view may have an impact on the data. To take this fact correctly into account, the estimated object has to be spatially larger than the observed field of view. At least half of the PSF support must be added on each side of the observed field of view to form the restored field of view.

The level of priors introduced in the restoration is balanced by hyper-parameters $\alpha$ and $\beta$ that are estimated by trial and error. The restored data cube $\boldsymbol{x}^{+}$is the solution of Eq. (3.1). It requires the minimization of the cost function $f(\boldsymbol{x})$ that involves a large number of parameters $\left(>10^{6}\right)$ with positivity constraints. To that end, we use the VMLM-B algorithm (Thiébaut, 2002) which is a limited memory variant of the variable metric method with BFGS updates (Nocedal and Wright, 1999). This algorithm has proved its effectiveness for image reconstruction and only requires the computation of the penalty function being minimized together with its gradient. The memory requirement is a few times the size of the problem.

\section{Results}

The quality of the presented algorithm was assessed on data from the MUSE IFS simulator. This data is a part of $51 \times 36$ spaxels (pixels size: $0^{\prime \prime} 2 \times 0^{\prime \prime} 2$ ) of the whole MUSE data cube. It contains 3463 spectral channels comprised between $480 \mathrm{~nm}$ and $930 \mathrm{~nm}$. The PSF was computed for a seeing of $1.1^{\prime \prime}$. This PSF shown on Fig. 1 is supposed to be separable and composed of a spatial field spread function (FSF) and a spectral line spread function (LSF). As shown in Fig. 1, both of them vary spectrally. FSF is Gaussian with a full width at half maximum that varies from $0^{\prime \prime} 75$ (3.75 pixels) at the red end to $0^{\prime \prime} 92$ (4.6 pixels) at the blue end. In addition, the MUSE IFS simulator provided a cube of the variance for each pixel as it will be estimated by the data reduction software of the instrument.

To perform the restoration, we first have to build the fast approximation of the operator $\mathbf{H}$ as defined Eq. (2.11). For these experiments, the PSF was sampled on a grid of $P=350$ evenly spaced wavelengths to give the $h_{p}$ and linear interpolation along the spectral dimension that was used for the weights $\phi_{p}$. As linear interpolation is used, the PSF $h_{\ell}$ centered on spectral channel $\ell$ with $\lambda_{p} \leq \lambda_{\ell} \leq \lambda_{p+1}$ is interpoled only using $h_{p}$ and $h_{p+1}$. The Euclidean norm of the differences between 


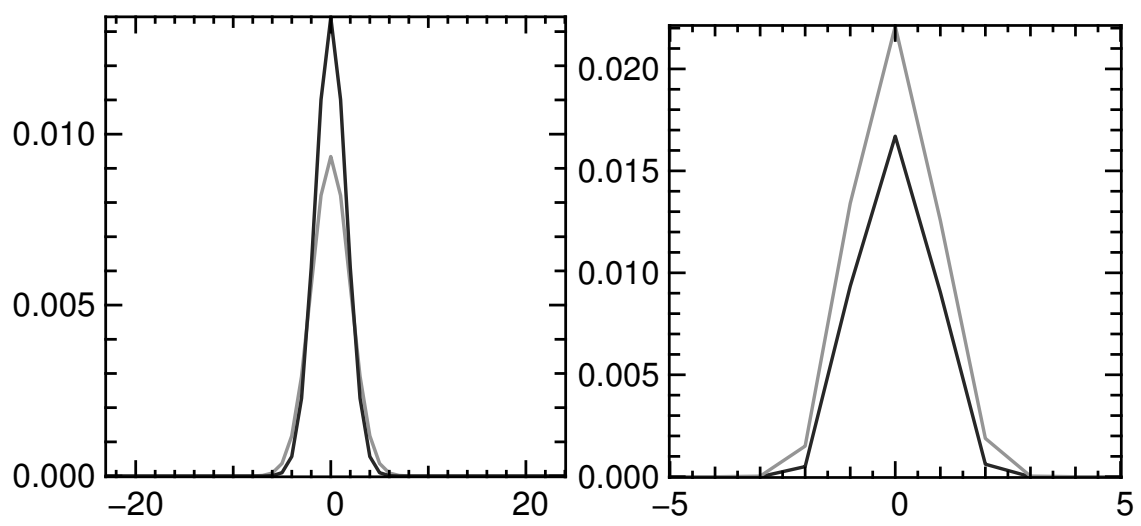

Fig. 1. Left: Profile of the PSF along a spatial dimension. Right: profile of the PSF along the spectral dimension. Grey and black profile correspond to the blue and the red ends of the IFS respectively.

the true PSF $h_{\lambda}$ and our approximation is less than $8 \times 10^{-5}(0.08 \%$ relatively to the euclidean norm of the PSF). That gives a quantitative estimate of the good quality of our approximation.

As stated in section 3.1, the restored field of view must be larger than the data FOV. In the presented experiments, the size of the restored FOV is extended to $64 \times 48$ spaxels and 3481 wavelengths.

The data were processed with both quadratic and spatial sparsity regularizations. The effectiveness is qualitatively evaluated by visual inspection and quantitatively by the root mean square error (RMSE):

$$
\operatorname{RMSE}(\boldsymbol{x})=\sqrt{\frac{1}{N_{\Omega} N_{\lambda}} \sum_{\boldsymbol{k}, \ell}[\boldsymbol{x}-\boldsymbol{o}]_{\boldsymbol{k}, \ell}^{2}},
$$

with $\boldsymbol{o}$ the truth. In both cases, the hyper-parameters $\alpha$ and $\beta$ were set to minimize the RMSE. For the quadratic case, with the hyper-parameters $\alpha=1$ and $\beta=1$, the algorithm converged in about 5 hours to the solution $\boldsymbol{x}_{\text {quad }}^{+}$with $\operatorname{RMSE}\left(\boldsymbol{x}_{\text {quad }}^{+}\right)=$ 0.418 . For the spatial sparsity case, the algorithm converged in about 8 hours to the solution $\boldsymbol{x}_{\text {Spar }}^{+}$with $\operatorname{RMSE}\left(\boldsymbol{x}_{\text {Spar }}^{+}\right)=0.344$ with the hyper-parameters $\alpha=15000$, $\beta=0.05$ and $\zeta=1$.

The results are shown on figures 2 and 3. Fig. 2 shows the data, the results and the true object integrated over the whole spectral range of the instrument. It clearly illustrates the gain in term of spatial resolution provided by our method. Both the shapes of the central galaxy and of the one near the upper left corner are recovered. Compared to the solution with spatial sparsity regularization $\boldsymbol{x}_{\mathrm{Spar}}^{+}$, the solution with quadratic regularization $\boldsymbol{x}_{\text {quad }}^{+}$shows more artifacts (e.g. on the bottom left part of the central galaxy) and bright spots are a bit over-smoothed.

We display in Fig. 3 spectral cuts through the heart of the central galaxy materialized by the dashed line in 2 . These figures show $(\theta, \lambda)$ images zoomed between 

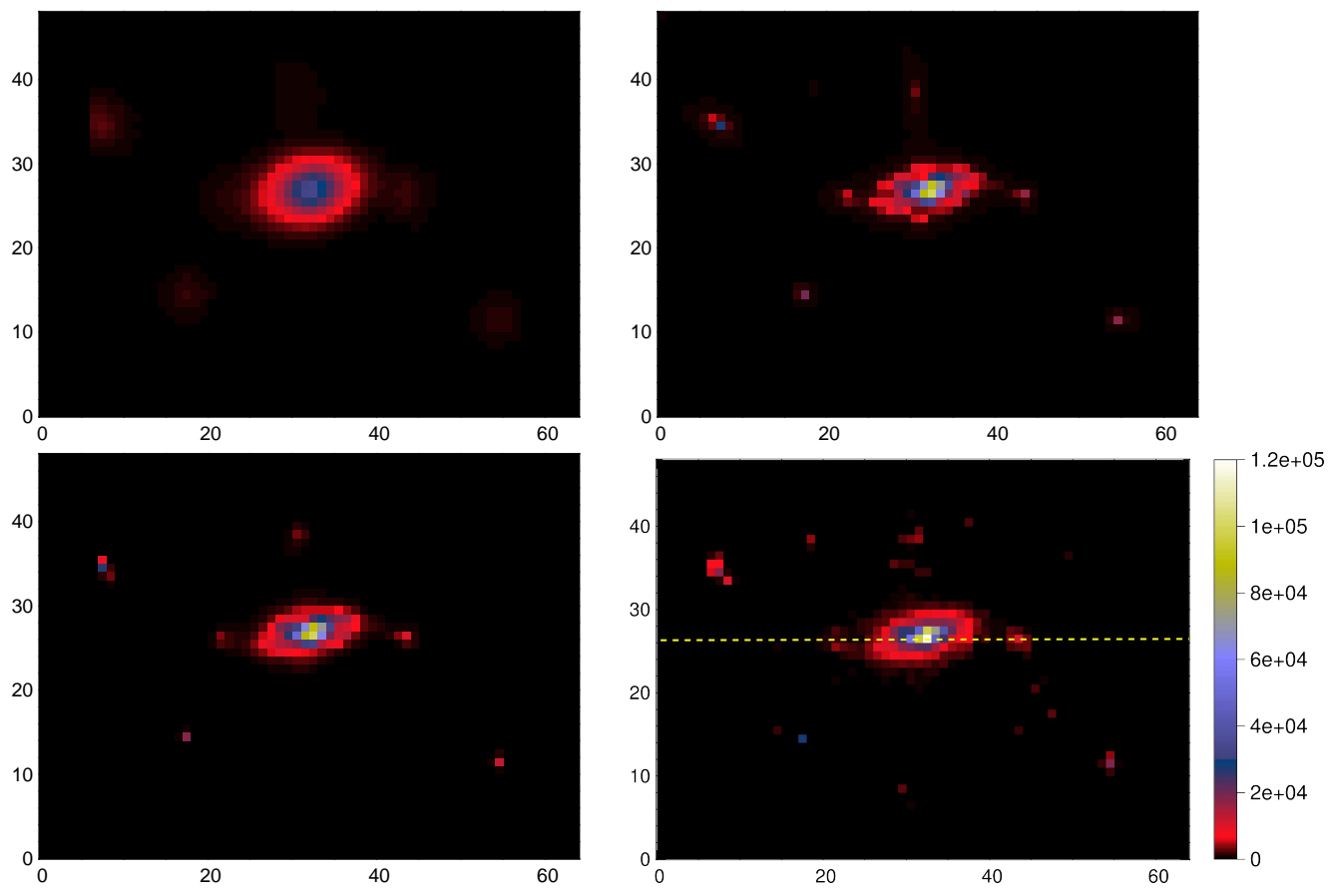

Fig. 2. Images spectrally integrated. Top left: raw data. Top right: restored object $\boldsymbol{x}_{\text {quad }}^{+}$ with quadratic regularization. Bottom left: restored object $\boldsymbol{x}_{\text {Spar }}^{+}$with spatial sparsity regularization. Bottom right: true object $\boldsymbol{o}$.

$567 \mathrm{~nm}$ and $574 \mathrm{~nm}$ for the data, both restorations and the true object. These plots show the resolution gain provided by our algorithm: the two brightest objects are well separated, with the spectrum at 43rd column visible in the restoration that was not visible in the data. Once again, the solution with quadratic regularization $\boldsymbol{x}_{\text {quad }}^{+}$shows much more artifacts (e.g. on the bright emission line at $\lambda=573 \mathrm{~nm}$ and $\theta=22$ ). Furthermore, the noise has been drastically reduced by our method as this can clearly be seen by looking at the background.

Figure 4 displays the spectra of the brighter spaxel of the galaxy $(\boldsymbol{\theta}=(33,27))$ of the data, the quadratic restoration $\boldsymbol{x}_{\text {quad }}^{+}$and the spatial sparsity restoration $\boldsymbol{x}_{\text {Spar }}^{+}$and the ground truth. Even though regularizations introduce some expected bias, the restored spectra are closer to the ground truth and far less noisy than the measured spectrum. In the spatial sparsity restoration $\boldsymbol{x}_{\mathrm{Spar}}^{+}$(red), most of the spectral features are preserved. These features are over-smoothed in the quadratic restoration $\boldsymbol{x}_{\text {quad }}^{+}$(green). The bias between restoration and is quite strong as it is the spectrum of the spaxel with the higher dynamical range and it tends to be flattened by the regularization. The hyper-parameters were tuned to provide the minimal RMSE for the whole field of view. As a consequence, the hyperparameters setting for a sufficient regularization of the faint sources is strong for 

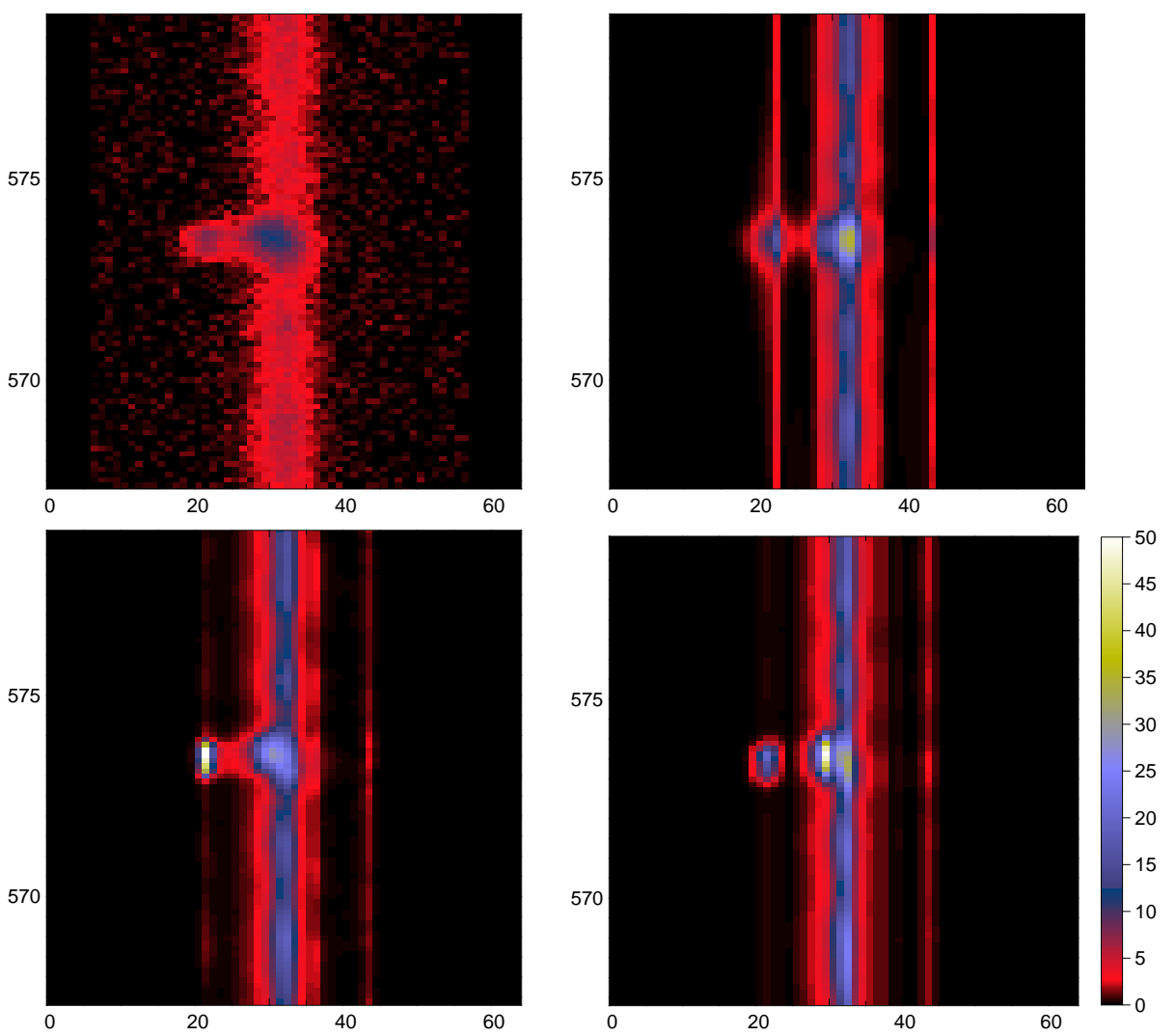

Fig. 3. $(\theta, \lambda)$ images of the cut materialized by the yellow line in Fig. 2 magnified between $567 \mathrm{~nm}$ and $574 \mathrm{~nm}$. Top left: raw data. Top right: restored object $\boldsymbol{x}_{\text {quad }}^{+}$ with quadratic regularization. Bottom left: restored object $\boldsymbol{x}_{\mathrm{Spar}}^{+}$with spatial sparsity regularization. Top right: true object $\boldsymbol{o}$.

bright sources and tends to smooth them. However, this bias disappears if we integrate spatially on few spaxels as we show in Fig. 5 on the spectra of the central $3 \times 3$ region of the central galaxy. This means that the bias is mainly imputable to the remaining blur.

\section{Conclusion}

In this paper, we present a method for restoring hyperspectral data. We especially focused on two points: (i) the design of an efficient operator modelling spectrally varying blur and (ii) a comparison between quadratic and spatial sparsity regularization functions.

We have shown that using PSF interpolation it is possible to design an effective operator approximating spectrally varying blur. Our formulation preserves 


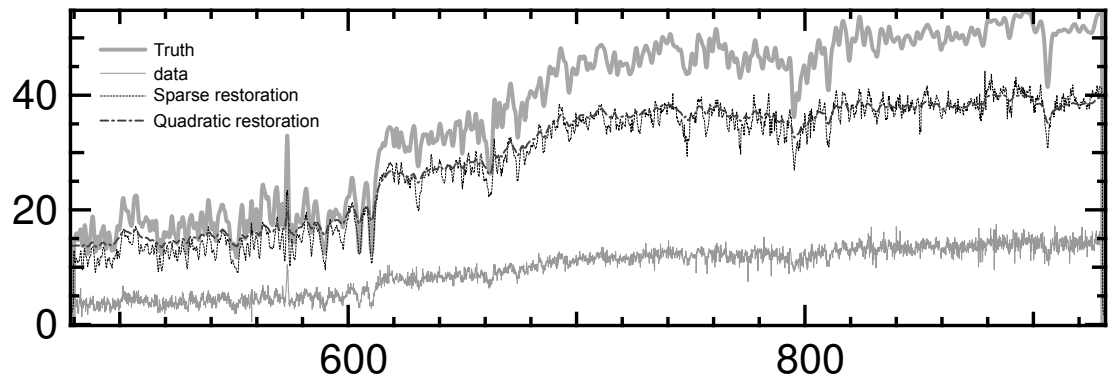

Fig. 4. Spectra of the brighter spaxel of the central galaxy of the data (thin grey line), the quadratic restoration $\boldsymbol{x}_{\text {quad }}^{+}$(dark grey dash dotted line) and the spatial sparsity restoration $\boldsymbol{x}_{\text {Spar }}^{+}$(thin dashed black line) compared to the true spectrum (thick grey line).

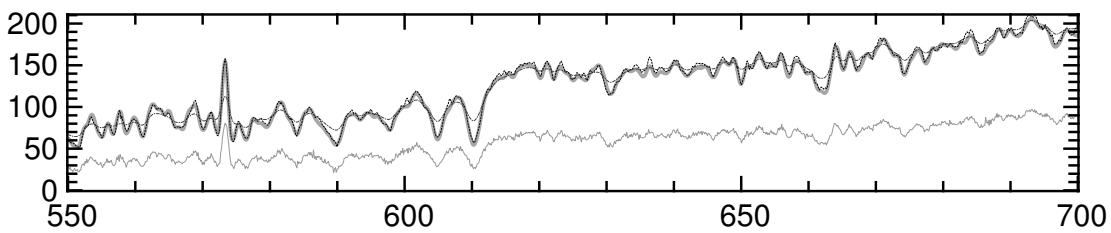

Fig. 5. Details (550 to $700 \mathrm{~nm}$ ) of spectra integrated on a $3 \times 3$ region centered on the brighter spaxel of the central galaxy of the data (thin grey line), the quadratic restoration $\boldsymbol{x}_{\text {quad }}^{+}\left(\right.$dark grey dash dotted line) and the spatial sparsity restoration $\boldsymbol{x}_{\text {Spar }}^{+}$(thin dashed black line) compared to the true spectrum (thick grey line).

the positivity, the normalization and the symmetry of the PSF. The computational cost of such approximation, that is twice as much as spectrally invariant convolution, remains tractable and it is possible to consider the processing of whole MUSE data cubes (size: $300 \times 300 \times 3463$ ) with nowadays CPU power. Furthermore, this type of operator can be easily extended to blurs that vary both spatially and spectrally as in wide field observations with adaptive optics.

By exploiting jointly spatial and spectral correlations present in the data, our method provides a strong spatial resolution enhancement and an effective denoising along the spectral dimension. Its deblurring performance is assessed on simulations showing the clear improvement in terms of both resolution and denoising. The comparison of a quadratic and a spatial sparsity regularization, shows that spatial sparsity regularization are less prone to artifacts and preserves most of the spatial and spectral features. However, the non linearity introduced by such regularization slows down the convergence of the optimization algorithm. In that case, optimization algorithms as Alternating Direction Method of Multiplier (ADMM) seem to provides faster convergence than our VMLMB algorithm as we shown in Thiébaut \& Soulez (2012).

This study as well as the one of Bourguignon et al.(2011a) show clearly the 
improvement given by a rigorous processing of hyperspectral astronomical data cube. However, two main problems remains in this field (i) the settings of the hyper-parameters, (ii) the estimation of the PSF. Our experience on SNIFS real data cube (Bongard et al., 2011) indicates that the hyper-parameters remains approximately identical for similar observations conditions. For the problems of the PSF estimation, we are currently studying blind deconvolution method where PSFs is estimated conjointly with the restoration only using the observations.

\section{Acknowledgments}

The authors would like to thank Roland Bacon P.I. of the MUSE instrument for providing the simulated data.

This work is supported by the French ANR (Agence Nationale de la Recherche), Éric Thiébaut and Loïc Denis work for the MiTiV project (Méthodes Inverses de Traitement en Imagerie du Vivant, ANR-09-EMER-008) and Ferréol Soulez is funded by the POLCA project (Percées astrophysiques grâce au traitement de données interférométriques polychromatiques, ANR-10-BLAN-0511).

Our algorithm has been implemented and tested with YORICK (http://yorick. sourceforge.net/) which is freely available.

\section{References}

Akgun, T., Altunbasak, Y., and Mersereau, R.: 2005, Image Processing, IEEE Transactions on 14(11), 1860

Benazza-Benyahia, A. and Pesquet, J. C.: 2006, in European Signal and Image Processing Conference, EUSIPCO'06, Vol. 5, pp 4-8, Firenze, Italy

Bertero, M. and Boccacci, P.: 1998, Introduction to Inverse Problems in Imaging, Taylor \& Francis

Bobin, J., Moudden, Y., Starck, J. L., and Fadili, J.: 2009, in Society of PhotoOptical Instrumentation Engineers (SPIE) Conference Series, Vol. 7446, p. 42

Bongard, S., Soulez, F., Thiébaut, É., and Pecontal, E.: 2011, Monthly Notices of the Royal Astronomical Society 418(1), 258

Bongard, S., Thiébaut, E., Soulez, F., and Pecontal, E.: 2009, in Proceedings of the First IEEE GRSS Workshop on Hyperspectral Image and Signal Processing - Evolution in Remote Sensing - (WHISPERS'09), cdrom, Grenoble France

Bourguignon, S., Mary, D., and Slezak, É.: 2011a, Statistical Methodology

Bourguignon, S., Mary, D., and Slezak, É.: 2011b, Selected Topics in Signal Processing, IEEE Journal of 5(5), 1002

Courbin, F., Magain, P., Kirkove, M., and Sohy, S.: 2000, The Astrophysical Journal 529(2), 1136

Denis, L., Thiébaut, E., and Soulez, F.: 2011, in 18th IEEE International Conference on Image Processing, pp 2873-2876, Bruxelles, France

Duijster, A., Scheunders, P., and Backer, S. D.: 2009, IEEE Transactions on Geoscience and Remote Sensing 47(11), 3892 
Fornasier, M. and Rauhut, H.: 2008, SIAM Journal on Numerical Analysis 46, 577

Galatsanos, N. and Chin, R.: 1989, IEEE Transactions on Acoustics, Speech, and Signal Processing 37(3), 415

Galatsanos, N., Katsaggelos, A., Chin, R., and Hillery, A.: 1991, IEEE Transactions on Signal Processing 39(10), 2222

Gaucel, J. M., Guillaume, M., and Bourennane, S.: 2006, in European Signal Processing Conference

Henault, F., Bacon, R., Bonneville, C., Boudon, D., Davies, R., Ferruit, P., Gilmore, G., Le Fevre, O., Lemonnier, J., and Lilly, S.: 2003, in Proceedings of SPIE, Vol. 4841, pp 1096-1107

Hunt, B. R. and Kubler, O.: 1984, IEEE transactions on acoustics, speech, and signal processing $\mathbf{3 2 ( 3 )}, 592$

Katsaggelos, A., Lay, K., and Galatsanos, N.: 1993, Image Processing, IEEE Transactions on $\mathbf{2}(\mathbf{3}), 417$

Kowalski, M. and Torrésani, B.: 2009, Signal, Image and Video Processing 3, 251

Lucy, L. and Walsh, J.: 2003, The Astronomical Journal 125(4), 2266

Mugnier, L., Fusco, T., and Conan, J.-M.: 2004, J. Opt. Soc. Am. A 21(10), 1841

Neelamani, R., Choi, H., and Baraniuk, R.: 2004, IEEE Trans. Signal Process. $\mathbf{5 2 ( 2 )}, 418$

Nocedal, J. and Wright, S.: 1999, Numerical optimization, Springer

Rodet, T., Orieux, F., Giovannelli, J., and Abergel, A.: 2008, IEEE Journal of Selected Topics in Signal Processing 2(5), 802

Soulez, F., Bongard, S., Thiébaut, E., and Bacon, R.: 2011, in Proceedings of the Third IEEE-GRSS Workshop on Hyperspectral Image and Signal Processing : Evolution in Remote Sensing,, cdrom, Lisbonne, Portugal

Soulez, F., Thiébaut, E., Gressard, A., Dauphin, R., and Bongard, S.: 2008, in Proceeding of the 16th European Signal Processing Conference EUSIPCO, Lausanne Suisse

Tekalp, M. and Pavlovic, G.: 1990, Signal Processing 19(3), 221

Thiébaut, E.: 2002, in J.-L. Starck (ed.), Astronomical Data Analysis II., Vol. 4847, pp $174-183$

Thiébaut, É. and Soulez, F.: 2012, in SPIE Astronomical Telescopes+ Instrumentation, 84451C, International Society for Optics and Photonics 